\title{
IDENTIDADES ACADÉMICAS EN CAMBIO EN EL SENO DE LA UNIVERSIDAD MULTILINGŮE
}

\section{Changing academic identities in the multilingual university}

\author{
Ane Larrinaga Renteria * \\ * Grupo de Investigación Parte Hartuz, UPV/EHU \\ ane.larrinaga@ehu.eus
}

\section{Palabras clave}

Identidades académicas Internacionalización de la universidad Cambio generacional Universidad del País Vasco Lengua vasca

\section{Keywords}

Academic identities Internationalization of the university Generational change University of the Basque Country Basque language

\section{Resumen}

En este artículo se presenta un análisis de algunas de las consecuencias que ha tenido la internacionalización de la universidad en la reformulación de las identidades profesionales de los académicos que desarrollan su actividad en una lengua minoritaria. La hipótesis de la que se parte es que los procesos globales de cambio que están modificando la educación superior afectan a los roles e identidades profesionales de los académicos. Más concretamente, se pretende mostrar que las recientes exigencias profesionales a las que se están adecuando los docentesinvestigadores comprenden también imperativos de orden lingüístico que van incorporándose progresivamente a las identidades cambiantes. El estudio empírico se ha llevado a cabo en un contexto universitario multilingüe con una lengua minoritaria, la Universidad del País Vasco, a través de entrevistas en profundidad que recogen las percepciones de docentes-investigadores vascoparlantes sobre su desempeño profesional. Este estudio, centrado especialmente en los componentes lingüísticos de la identidad profesional de los académicos, ha permitido identificar algunas tendencias de cambio reveladas a través del relevo generacional, que inciden en el desarrollo de la lengua minoritaria. En especial, la emergencia de fuertes orientaciones estratégicas en el diseño de las carreras académicas, la creciente centralidad de la identidad investigadora frente a la identidad docente, y la preeminencia de un ethos académico vinculado a las identidades disciplinares de las tecnologías y las ciencias experimentales.

\section{Abstract}

This article presents an analysis of some of the consequences that the internationalisation of the university has had on the reformulation of the professional identities of academics who develop their activity in a minority language. The starting hypothesis is that the global processes of change that are altering higher education are affecting the professional roles and identities of academics. More concretely, the article aims to show that the recent professional requirements to which lecturer-researchers are adapting also include imperatives of a linguistic type that are being progressively incorporated into changing identities. The empirical study was carried out in a multilingual university context with a minority language: the University of the Basque Country, and involved in-depth interviews that gathered the perceptions of Basque-speaking lecturerresearchers about their professional efforts. This study, which focused especially on the linguistic components of the academics' professional identity, made it possible to identify some tendencies of change revealed through generational change that have an impact on the development of the minority language. In particular, the emergence of strong strategic orientations in the design of academic careers, the growing centrality of the research identity against the teaching identity, and the pre-eminence of an academic ethos linked to the disciplinary identities of the technologies and the experimental sciences.

Larrinaga Renteria, A. (2016). Identidades académicas en cambio en el seno de la universidad multilingüe. Papeles del CEIC, vol. 2016/2, papel 163, CEIC (Centro de Estudios sobre la Identidad Colectiva), Universidad del País Vasco, http://dx.doi.org/10.1387/pceic.15978

Recibido: 2/2016; Aceptado: 6/2016 


\section{Presentación}

Los procesos de internacionalización en el ámbito europeo han traído consigo transformaciones que afectan a la organización, las funciones y la lógica de funcionamiento de la universidad actual, a sus valores y actividades (docencia e investigación). Esta profunda metamorfosis institucional compromete también los modelos de desempeño de los roles profesionales de docentes e investigadores, y la forma de comprender la constitución de sus identidades académicas. Así, a medida que se desarrollan nuevos requerimientos y exigencias institucionales, van emergiendo identidades académicas renovadas y reformuladas. Entre los múltiples requerimientos profesionales de la universidad internacionalizada destacan los de orden lingüístico.

En efecto, como consecuencia de los procesos de globalización e internacionalización, en el siglo XXI las universidades del EEES (Espacio Europeo de Educación Superior) han comenzado a transitar desde sistemas eminentemente monolingües, basados en la lengua oficial del país, a otros bilingües o multilingües (Lasagabaster, 2012b). Sin embargo, tal transición lingüistica no se produce siempre bajo las mismas condiciones. Un caso lingüístico especial lo constituyen los ámbitos de educación superior en los que la lengua estatal coexiste con una lengua minoritaria.

El interés por el estudio de este contexto lingüistico particular nos ha llevado a centrar la investigación en la Universidad del País Vasco/Euskal Herriko Unibertsitatea (UPV/EHU). La UPV/EHU es una universidad bilingüe que se encuentra actualmente inmersa en procesos de cambio institucional derivados de la internacionalización de la educación superior, entre otros, la implantación del inglés como lingua franca, la extensión del multilingüismo en la investigación y la docencia, y la evaluación de la calidad y la competitividad económica. Las transformaciones en curso, así como su condición lingüistica particular, caracterizada por la coexistencia de dos lenguas (una estatal y otra socialmente minoritaria, pero institucionalmente promovida a través de políticas públicas) que la sitúan, junto con algunas universidades de Cataluña, en una posición singular en el contexto europeo, han originado la elección de esta universidad como escenario idóneo para el desarrollo de la investigación, para la que se propone un estudio de caso. El estudio que se presenta a continuación tiene por principal objetivo analizar las implicaciones lingüisticas del proceso actual de redefinición 
de las identidades académicas, examinando concretamente la incidencia de las identidades emergentes en las opciones lingüísticas de los académicos que trabajan en lengua minoritaria, en un contexto universitario crecientemente internacionalizado.

\section{LA INTERNACIONALIZACIÓN DE LA UNIVERSIDAD: IDENTIDADES PROFESIONALES Y CONDICIONES LINGÜÍSTICAS EN CAMBIO}

La universidad del siglo $\mathrm{XXI}$ está sometida a un intenso proceso de internacionalización. La internacionalización puede entenderse como el conjunto de programas y de políticas que los gobiernos y las universidades ponen en marcha para hacer frente a la globalización y a la masificación de la educación superior (Altbach, Reisberg y Rumbley, 2009). Las exigencias de la globalización económica y los imperativos que plantea la nueva economía basada en el conocimiento (Burton-Jones, 2001; Schleider, 2006) han estado presentes en las declaraciones y documentos que se han elaborado para la creación del Espacio Europeo de Educación Superior; y, de manera muy manifiesta, en el plan de Bolonia y en el proyecto diseñado en el Consejo Europeo de Lisboa en el 2000, que marcaba el objetivo estratégico de convertir a Europa en la economía del conocimiento más competitiva y dinámica del mundo. También en las conclusiones del Consejo de Europa sobre el marco estratégico para la cooperación europea en educación y formación, conocido como "ET 2020" (The Council of the European Union, 2009). En este escenario, en el que el conocimiento se ha convertido en la forma más importante de capital global, se está produciendo un cambio en las funciones de la universidad (Canaan y Shumar, 2008). Justamente, la internacionalización surge como una estrategia de las universidades para diferenciarse dentro de un sector universitario que es concurrencial y estratégico en materia de desarrollo económico, empleo e inversión.

Como todo gran proceso de cambio social, el proceso de internacionalización de la universidad no ha estado exento de incertidumbres y dificultades. Definitivamente, la internacionalización ha traído consigo una gran mutación institucional, organizativa y cultural al campo de la educación superior; también innumerables tensiones y contradicciones (Ninnes y Hellstén, 2011). Entre las múltiples dimensiones que comprende este complejo proceso de cambio se encuentran las que afectan a los roles e identidades profesionales desempeñados por docentes e investigadores (Barnett y Di Napoli, 2008; Gordon y Whitchurch, 
2010; Kehm y Teichler, 2013), y también a las prácticas lingüísticas que lleva incorporadas su desempeño profesional. El cambio que está viviendo la profesión docente-investigadora en las actuales universidades puede comprenderse en el marco de la tendencia general de decadencia de los programas institucionales diseñados en la modernidad, descrita por ciertos análisis (Bauman, 2004; Dubet, 2006). Según éstos, asistimos al fin de aquellos procesos sociales que hasta ahora habian transformado valores y principios definidos en las instituciones educativas en acción y en subjetividad, a través de un trabajo profesional específico y organizado; tales procesos permitían hasta épocas recientes la coherencia e identidad entre actor y sistema, entre institución y roles (Dubet, 2006, 2009).

En la actualidad, las prácticas académicas se alejan paulatinamente del programa de universidad vigente durante el siglo $X X$, se impone la heterogeneidad de valores y principios, se disuelve la unidad de las finalidades imperantes anteriormente, y los docentes se ven sometidos a demandas diversas y a lógicas de acción cambiantes y, en ocasiones, contrapuestas. En estas condiciones, no siempre coinciden las identidades profesionales definidas desde los imperativos y necesidades de las instituciones terciarias y los sentidos que los propios académicos atribuyen a su trabajo (Churchman y King, 2009). En el mundo académico proliferan las reflexiones sobre lo que debe ser la universidad, y sobre el trabajo académico y su metamorfosis en las nuevas condiciones (Michavila y Martínez, 2004; Montané y Sánchez de Serdio, 2011; Hernández, DelgadoGal y Pericay, 2013). Los académicos deben resituarse en múltiples racionalidades, las de la cultura y la producción, entre otras, y en múltiples identidades, la académica y la investigadora (Villarruel, 2012), la cultural y la gerencial (Di Napoli, 2010), la local y la global (Michavila, 2012). En la línea de lo sugerido por Dubet, en la práctica se produce un desplazamiento de lo que era la legitimidad de la institución a una legitimidad racional, fundada en la eficacia del trabajo efectuado y en la certificación constante, una transferencia de los argumentos de legitimación desde un registro vocacional a un registro técnico, con procedimientos de evaluación y de control dirigidos a demostrar la eficacia del trabajo efectuado (Dubet, 2006). Pero, las nuevas identidades no responden ya a las exigencias internas del campo académico en el que se desarrollan, sino a imperativos externos al mismo que provienen, cada vez con mayor intensidad, de los mercados económicos. 
La universidad actual sucumbe a la penetración de la lógica económica. En este sentido, la transferencia de modelos empresariales, tanto en los modos de gestión como en los valores, prioridades y lógicas de funcionamiento de las instituciones de la educación superior, ha supuesto la aplicación de métodos gerencialistas que eran propios del sector privado (Marginson y Considine, 2000), como la planificación por objetivos, los recursos humanos y la supervisión de resultados y responsabilidad; estos últimos incluyen los resultados del personal docente y los consiguientes sistemas de recompensas simbólicas y económicas ligados a los nuevos parámetros de calidad (Ball y Youdell, 2007; Altbach, Reisberg y Rumbley, 2009). La competencia por los recursos y la excelencia entre universidades lleva aparejada un mayor control sobre sus profesionales a partir de la regulación de las dimensiones evaluables de las tareas docentes y de investigación, (Montané y Sánchez de Serdio, 2011). De forma paralela, se ha ido instalando un proceso de auto-regulación del propio personal docente e investigador en términos de productividad y eficiencia; de este modo, en la construcción de identidades profesionales competitivas y flexibles (Sennet, 1998) se han antepuesto aquellas actividades y prácticas que son positivamente recompensadas y que constituyen el salvoconducto para la promoción en la carrera académica.

Por consiguiente, nuevas jerarquías simbólicas se han instalado en el campo de la educación superior, produciéndose una reversión de los valores académicos tradicionales, -cuyo resultado más visible es la devaluación de la docencia frente a la investigación-, (Sancho, Creus y Padilla, 2010; Faure, Soulié y Millet, 2011) y, en general, ha arraigado en la institución una cultura caracterizada por valores instrumentales que, progresivamente, devienen centrales en la definición de las nuevas identidades profesionales (Harris, 2005). Así, la racionalidad instrumental, que ha sustituido a la ética profesional que orientaba el proceso de toma de decisiones (Shapper y Mayson, 2011), guía actualmente la readaptación de las identidades profesionales a las condiciones crecientemente mercantilizadas del mundo académico (Di Napoli, 2010). Según las lecturas más críticas a este proceso, el gerencialismo y la cultura de la eficiencia han erosionado la autonomía de los docentes-investigadores, han fragmentado y "taylorizado" sus actividades profesionales y han alienado a los académicos de la estructura de toma de decisiones dentro de las universidades (Shapper y Mayson, 2011), subordinando su función a los intereses estratégicos de la institución y provocando su 
desprofesionalización progresiva (Altbach, Risberg y Rumbley, 2009). Tales cambios parecen reflejarse de forma más evidente en las nuevas generaciones de académicos, aunque entre éstos también se desarrollan a menudo estrategias de resistencia y prácticas de negociación frente a las presiones institucionales (Archer, 2008).

Dentro de esta mutación profesional que es de carácter multidimensional, se incluyen los componentes lingüísticos necesariamente implicados en el desempeño de la actividad profesional. En efecto, el logos instrumental del modelo gerencialista fija también, de manera implícita, el valor simbólico y el grado de legitimidad de las prácticas lingüisticas a través de las que se materializa la actividad profesional, sea en la docencia, sea en la actividad investigadora. Tales prácticas vienen cada vez más determinadas por los indicadores del rendimiento universitario, que fijan los patrones internacionales de calidad y excelencia (Gazzola, 2012). En este contexto, la internacionalización de la educación superior impulsa la consolidación del multilingüismo en el ámbito universitario.

En la dimensión docente, el paradigma dominante del multilingüismo ha traído consigo una reorientación del rol académico en los contextos que eran hasta ahora bilingües, en la medida en que el inglés ha pasado a compartir currículo con las lenguas oficiales - tanto estatales como minoritarias-. En los contextos universitarios con lengua minoritaria, el proceso de introducción del inglés en la docencia no está exento de tensiones y ambigüedades, y aparecen voces discrepantes ante la potencial reducción del espacio de las lenguas minoritarias (Lasagabaster, 2012a). Existen investigaciones que indican que la introducción de la tercera lengua como medio de instrucción en este tipo de universidades (en Cataluña y en el País Vasco, concretamente) provoca en los alumnos y profesores cuya primera lengua es la lengua minoritaria una actitud más reticente que en los mismos grupos cuya primera lengua es la estatal (Cots, 2013; Doiz, Lasagabaster y Sierra, 2013), aunque el alumnado percibe cada vez más la introducción de la tercera lengua como un recurso económico beneficioso para la empleabilidad (Sabaté-Dalmau, 2016). Ciertamente, los contextos bilingües representan situaciones sociolingüisticas particulares en las que la introducción de la lengua franca puede afectar la ecología lingüistica de la institución, debido a la vulnerabilidad de los equilibrios entre las lenguas, y a la posición asimétrica de la lengua minoritaria (Cots, Llurdá y Garrett, 2014; Lasagabaster, 2012c). En estos frágiles equilibrios 
está implicada, como no podía ser de otra manera, la ordenación de la actividad profesional de los docentes en lengua minoritaria.

En efecto, en las universidades con lengua minoritaria que transitan desde el bilingüismo hacia el multilingüismo, el multilingüismo adquiere sentidos y objetivos muy diversos: por un lado, implica la promoción de la lengua minoritaria junto a la lengua estatal $y$, por otro, el impulso del conocimiento y uso del inglés, así como la atracción de estudiantes extranjeros. Todos estos sentidos se han intentado armonizar desde los discursos institucionales (Alcón, 2012; Cots, Lasagabaster y Garrett, 2012). Pero, estos objetivos desencadenan, en la práctica, fuerzas divergentes que determinan formulaciones contradictorias y superpuestas del rol docente. Como muestran algunos estudios, en las universidades con políticas de promoción de lenguas minoritarias se constata la existencia de condiciones particulares que rigen la actividad docente en lengua minoritaria - a menudo, mayor número de asignaturas para impartir por el académico, carga adicional de créditos docentes, ausencia de material docente en lengua minoritaria, necesidad de producir textos en una lengua que no está normalizada y que requiere modernización del lenguaje científico (Cenoz, 2012; Iglesias y Salaburu, 2003)- En tales condiciones, el desarrollo integral de la actividad docente aparece idealmente asociado a un ethos profesional alejado de la racionalidad instrumental y próximo, en cambio, a la idea de compromiso de la universidad hacia la comunidad local y a la tradición de servicio público, valores éticos que han guiado tradicionalmente el funcionamiento del mundo universitario (Altbach, Reisberg y Rumbley, 2009).

Pero, la promoción, incentivación y reconocimiento académico de prácticas lingüísticas en lenguas mejor posicionadas social $y$ académicamente debilitan, de facto, ese patrón de identidad profesional, que aparece cada vez más devaluado en el mercado universitario de los bienes simbólicos, de los reconocimientos y de las recompensas económicas. Así, aunque institucionalmente el discurso del multilingüismo asigna a las lenguas minoritarias un papel predominantemente docente en la dialéctica global-local - junto a la lengua estatal y el inglés- (Michavila, 2012; Alcón, 2012; Lasagabaster, 2012b), las identidades académicas promovidas por la internacionalización lingüística actual bajo el principio del multilingüismo se muestran alejadas de aquellas tradiciones profesionales que han sostenido hasta ahora a las lenguas minoritarias en su papel de lengua vehicular en la educación superior. 
Es en la actividad investigadora donde los criterios de gestión del rendimiento impuestos por la internacionalización hegemónica, sus indicadores de evaluación y su consiguiente estructura implícita de incentivos económicos y simbólicos, han impuesto con mayor intensidad su lógica y han reordenado el campo de jerarquías científicas y de identidades académicas en detrimento de la diversidad lingüistica. Aún tomando en cuenta que en las actividades profesionales cotidianas de investigación la lengua minoritaria y la lengua estatal pueden tener hoy en día una presencia relativa en las relaciones personales y de gestión (Vila, Bretxa y Comajoan, 2014), la proyección científica (producción de artículos, comunicación de resultados de investigación, etc.) se materializa mayoritariamente en inglés. En este sentido, la calidad de la investigación científica se ha desplazado del proceso investigador, tomado en sentido estricto, al proceso de comunicación de los resultados, de tal manera que la calidad de las investigaciones y de los investigadores se mide actualmente en términos de publicación en revistas de alto impacto en lengua inglesa (Gutiérrez-Solana, Ruiz y Etayo, 2012; Hamel, 2013). Las bases de datos que suministran los factores de impacto y los sistemas de indicadores bibliométricos internacionalmente vigentes revelan la abrumadora preeminencia de las publicaciones en inglés frente a otras lenguas, y la supremacía de los países anglófonos -encabezados por los EE.UU.- en los rankings de producción científica (Truchot, 2010; Lynch, 2011; Hamel, 2013). Esto ha generado no sólo una situación de distribución sesgada de las lenguas en las publicaciones indexadas (Gazzola, 2012; Ammon, 2012; Hamel, 2013) sino también la instauración de una lógica implícita de gratificaciones que, de facto, orienta de forma estratégica las opciones lingüísticas en la actividad investigadora de los académicos.

La falta de instrumentos bibliométricos adecuados y de agencias de evaluación que apliquen criterios de carácter local junto a otros globales para la evaluación de la calidad e impacto de las publicaciones afecta especialmente a la actividad de los académicos en lenguas minoritarias (Larrinaga, 2007; Artetxe, 2011), y dificulta la publicación científica, e incluso divulgativa, en estas lenguas (Cenoz, 2012), devaluando la actividad que se realiza a través de ellas. La consecuencia potencial es un brain drain lingüístico, que lleva a los académicos a renunciar a la publicación en lenguas minoritarias (Artetxe, 2011). De este modo, puede decirse que el valor simbólico de las lenguas minoritarias en el campo de la educación superior decrece en la misma medida en que aumentan las preferencias de 
los académicos hacia la investigación, como consecuencia de las nuevas jerarquías simbólicas establecidas en las tareas propias del rol académico -con el predominio de la investigación sobre la docencia (Faure, Soulié y Millet, 2011) - y de las implícitas condiciones lingüísticas de su materialización.

\section{NOTAS METOdOLÓGICAS}

El estudio de caso se ha llevado a cabo en una universidad multilingüe, la Universidad del País Vasco/Euskal Herriko Unibertsitatea (UPV/EHU), en la que se imparte docencia fundamentalmente en dos lenguas oficiales, el español y el euskara además de otras lenguas extranjeras (sobre todo el inglés). Situada en la Comunidad Autónoma Vasca, la UPV/EHU es la universidad pública más importante del ámbito de la comunidad vascoparlante, tanto desde el punto de vista de los recursos humanos como por su producción científica y su capacidad investigadora. Según los estatutos aprobados en 1984, la UPV/EHU se define como una universidad bilingüe, con dos lenguas oficiales, el vasco o euskara (lengua minoritaria) y el castellano (lengua estatal, mayoritaria). Los estatutos actualizados en 2011 confirman el carácter bilingüe y agregan que la universidad impulsará el conocimiento de otras lenguas en las que se desarrolla la ciencia (UPV/EHU, 2011). La mayor parte de los créditos obligatorios se ofertan actualmente en euskara y en castellano, pero el euskara tiene una presencia menor en algunas áreas y en los créditos optativos de grado $y$, especialmente, en los estudios de posgrado.

Conforme a los datos de la propia universidad correspondientes al año 2012-13, son más de 50.000 los estudiantes matriculados en dicho curso, y en la misma fecha el Personal Docente e Investigador (sin incluir el personal únicamente investigador) asciende a unas 4.600 personas; de este grupo, aproximadamente un $43 \%$ son bilingües euskara-castellano (UPV/EHU, 2013a). En el año 2006 se supera el 50\% de estudiantes que realizan la selectividad en euskara, llegando el alumnado bilingüe a constituir el $45 \%$ de las nuevas matrículas de la UPV/EHU ya en el curso 2007/08 (Cenoz, 2012). A esta progresión del bilingüismo han venido a sumarse sucesivos Planes de Plurilingüismo que, aprobados desde el año 2005, han promovido la utilización de lenguas extranjeras, y en particular del inglés como lengua vehicular en la UPV/EHU. Actualmente los créditos ofertados en euskara en los estudios de grado constituyen poco más del $80 \%$ de los créditos totales de dichos grados, en tanto que en español se 
ofertan casi el $90 \%$, y en otras lenguas (fundamentalmente en inglés) el $7 \%$. En el año 2012, un 43\% de los libros editados por la UPV/EHU lo fueron en euskara (incluyendo traducciones de manuales, tesis doctorales y otros materiales docentes), el $55 \%$ en español y el $2 \%$ en otras lenguas (UPV/EHU, 2013b).

El propósito del estudio desarrollado en la UPV/EHU ha sido la detección e identificación de diferentes discursos producidos por docentes e investigadores vascoparlantes en torno a actitudes y representaciones de la lengua minoritaria en la universidad, en el contexto de los nuevos requerimientos profesionales derivados de los procesos de internacionalización del campo de la educación superior. Se ha tratado de determinar si existen variaciones generacionales tanto en las percepciones de estos académicos sobre la conformación de sus identidades profesionales, como en las estrategias lingüísticas desarrolladas por aquellos, con el fin de descubrir el impacto potencial de los procesos de cambio global en las oportunidades de desarrollo de las lenguas minoritarias incorporadas a la universidad. Acorde a los objetivos del trabajo, el estudio se ha realizado desde una perspectiva metodológica cualitativa. En total se han llevado a cabo 25 entrevistas en profundidad. La recogida de datos se ha materializado mediante la técnica de la entrevista en profundidad semiestructurada, puesto que se ha buscado la focalización de los discursos de las personas entrevistadas en determinados temas asociados al objeto de investigación. El guión general de la entrevista se ha organizado en torno a cuatro bloques temáticos: (1) ámbitos de socialización y trayectoria vital, (2) experiencias profesionales en la universidad bilingüe, (3) diseño y representación del itinerario académico-profesional y (4) percepción de las funciones de la lengua minoritaria y de las lenguas en contacto dentro de los cambios institucionales que vive la universidad.

Dados los objetivos del estudio, en el diseño de la muestra se ha considerado una variable significativa la edad-generación de las personas entrevistadas. Siguiendo la perspectiva del cambio social aprehendido a través del relevo generacional, la selección de las unidades que integran la muestra del presente trabajo (compuesta por docentes investigadores vascoparlantes de la UPV/EHU) se ha realizado tomando en consideración, primeramente, el criterio generacional. Han sido establecidos para la investigación dos períodos generacionales que se han considerado relevantes para el estudio. Por un lado, se han seleccionado 10 docentes 
investigadores/as de una primera generación; más allá del concepto biológico, esta generación se ha definido y delimitado para el análisis tomando como base a académicos que fueron contratados como docentes en las décadas de 1970 y 1980, etapa en la que el uso de la lengua vasca carecía de regulación y promoción institucional en la UPV/EHU. Según las características requeridas en el diseño estratégico de la investigación, los sujetos seleccionados fueron precursores en la implantación de la enseñanza en euskara en la UPV/EHU en un lapso de tiempo en que tal lengua no aparecía reconocida como lengua oficial en los estatutos de la universidad, $y$, en consecuencia, no se habian desarrollado programas institucionales para su desarrollo. Desde el punto de vista social, este período coincide con la primera etapa posfranquista y con el progresivo acceso al espacio público y a la educación formal de lenguas minoritarias del estado español, como el euskara, que habian estado socialmente relegadas y políticamente prohibidas.

Por otro lado, han sido seleccionados 15 docentes investigadores/as de una segunda generación, que fueron contratados/as expresamente para impartir docencia en euskara en las décadas de 1990 y 2000, etapa en la que la docencia en esta lengua aparece ya regulada y promovida institucionalmente en la UPV/EHU. Las características lingüisticas requeridas a los componentes de este segundo colectivo generacional han sido, de un lado, la de ser sujetos - al menos- bilingües (euskara y castellano) y, de otro, la de impartir una parte o la totalidad de su docencia en lengua vasca. Este segundo periodo viene caracterizado en la educación superior por una intensificación de las dinámicas de la globalización, tanto en su dimensión lingüística (establecimiento de programas de multilingüismo) como profesional (sistemas de evaluación y acreditación del profesorado, sistemas de movilidad, etc.) y organizativa-gerencial. A nivel social, coincide con la progresiva institucionalización y consolidación de políticas lingüísticas de promoción del euskara en el contexto autonómico, especialmente en la Comunidad Autónoma Vasca (CAV). El colectivo de docentes incluidos en lo que hemos llamado "primera generación" tiene en el momento de la investigación un promedio de edad de 55,8 años, y el colectivo de la "segunda generación" un promedio de 37,06 años.

Con el objetivo de obtener la mayor diversidad posible en los discursos, también se han tomado en cuenta otras variables para completar los perfiles de las personas entrevistadas. La primera variable ha sido el área 
de conocimiento de los/as docentes investigadores/as: 7 académicos/as son de CC. Experimentales, 3 de Tecnología, 7 de CC. Sociales y Jurídicas, 4 de CC. de la Salud y 4 de CC. Humanas. La segunda ha sido la posesión o ausencia de publicaciones en euskara: Sí (16), No (9). También se ha tomado en consideración el centro de adscripción: las personas entrevistadas están adscritas a 16 centros distintos, tanto facultades como escuelas universitarias y escuelas superiores de la UPV/EHU, y a los tres campus-es que componen dicha universidad. En la muestra se encuentran presentes la diversidad de jerarquías académicas. Por último, se ha buscado un equilibrio en el sexo-género de las personas entrevistadas: han sido entrevistados/as 14 docentes investigadores varones y 11 mujeres. Las variables consideradas para completar los perfiles de las personas entrevistadas, aparecen recogidas en la siguiente tabla:

\begin{tabular}{|c|c|c|c|c|c|}
\hline Sexo/Edad & Generación & $\begin{array}{c}\text { Publicación } \\
\text { Euskera }\end{array}$ & Campus & Area conoc. & Centro \\
\hline $\mathrm{V} /+60$ & 1 & $\mathrm{SI}$ & Bizkaia & Experiment. & E. Ingeniería \\
\hline V/53 & 1 & $\mathrm{SI}$ & Gipuzkoa & Tecnología & l.informática \\
\hline$M / 56$ & 1 & SI & Bizkaia & Experiment. & $\begin{array}{l}\text { Ciencia y } \\
\text { Tecnol. }\end{array}$ \\
\hline M / 59 & 1 & $\mathrm{SI}$ & Bizkaia & Experiment. & $\begin{array}{l}\text { Ciencia y } \\
\text { Tecnol. }\end{array}$ \\
\hline$V / 58$ & 1 & $\mathrm{SI}$ & Bizkaia & Experiment. & $\begin{array}{l}\text { Ciencia y } \\
\text { Tecnol. }\end{array}$ \\
\hline$M /+60$ & 1 & SI & Gipuzkoa & Humanas & $\begin{array}{l}\text { Educación, } \\
\text { Filos. }\end{array}$ \\
\hline $\mathrm{V} / 52$ & 1 & $\mathrm{SI}$ & Bizkaia & Experiment. & $\begin{array}{l}\text { Ciencia y } \\
\text { Tecnol. }\end{array}$ \\
\hline$M / 53$ & 1 & $\mathrm{SI}$ & Gipuzkoa & C. Salud & Psicología \\
\hline $\mathrm{V} / 49$ & 1 & $\mathrm{SI}$ & Araba & Humanas & Educación \\
\hline $\mathrm{V} / 57$ & 1 & NO & Gipuzkoa & Tecnología & $\begin{array}{l}\text { E. } \\
\text { Arquitectura }\end{array}$ \\
\hline $\mathrm{V} / 31$ & 2 & $\mathrm{SI}$ & Bizkaia & Experiment. & E. Ingeniería \\
\hline$M / 36$ & 2 & NO & Araba & C. Sociales & $\begin{array}{l}\text { Rel. Lab. } \\
\text { Trab.s }\end{array}$ \\
\hline $\mathrm{V} / 29$ & 2 & $\mathrm{SI}$ & Bizkaia & C. Sociales & $\begin{array}{l}\text { Economía y } \\
\text { Emp }\end{array}$ \\
\hline$M / 28$ & 2 & NO & Bizkaia & Experiment. & E. Ingeniería \\
\hline$V / 30$ & 2 & $\mathrm{SI}$ & Bizkaia & C. Salud & $\begin{array}{l}\text { Medicina y } \\
\text { Enf. }\end{array}$ \\
\hline $\mathrm{V} / 40$ & 2 & NO & Gipuzkoa & C. Sociales & Derecho \\
\hline $\mathrm{V} / 46$ & 2 & NO & Bizkaia & Humanas & Bellas Artes \\
\hline $\mathrm{V} / 42$ & 2 & SI & Araba & Tecnología & $\begin{array}{l}\text { EU } \\
\text { Ingeniería }\end{array}$ \\
\hline$M / 42$ & 2 & $\mathrm{SI}$ & Araba & Humanas & Letras \\
\hline$M / 28$ & 2 & $\mathrm{SI}$ & Araba & C. Salud & Farmacia \\
\hline
\end{tabular}




\begin{tabular}{|c|c|c|l|l|l|}
\hline M / 41 & 2 & NO & Bizkaia & C. Sociales & $\begin{array}{l}\text { Sociales y } \\
\text { Com }\end{array}$ \\
\hline M / 39 & 2 & SI & Bizkaia & C. Sociales & $\begin{array}{l}\text { Rel.Lab. } \\
\text { Trab. } \text { S }\end{array}$ \\
\hline V/43 & 2 & NO & Bizkaia & C. Sociales & $\begin{array}{l}\text { Sociales y } \\
\text { Com }\end{array}$ \\
\hline M / 29 & 2 & SI & Bizkaia & C. Sociales & $\begin{array}{l}\text { Sociales y } \\
\text { Com }\end{array}$ \\
\hline V/48 & 2 & SI & Bizkaia & C. Salud & $\begin{array}{l}\text { Medicina y } \\
\text { Enf }\end{array}$ \\
\hline
\end{tabular}

Tabla 1: Perfiles de los académicos de la UPV/EHU entrevistados.

\section{LA TRANSFORMACIÓN DE LAS IDENTIDADES ACADÉMICAS: LAS VOCES DE LOS DOCENTES-INVESTIGADORES VASCOPARLANTES DE LA UNIVERSIDAD DEL PAÍS VASCO}

El análisis de las entrevistas realizadas a académicos vascófonos de la UPV/EHU ha permitido reconocer varias tendencias de cambio en las identidades académicas, algunas de carácter global, relacionadas con las nuevas exigencias institucionales de la internacionalización a las que está sometida la construcción de la carrera académica hoy en día, y otras, que son fruto de la adaptación de los procesos generales a las condiciones lingüisticas particulares de una universidad con lengua minoritaria. La confluencia de unas y otras constituyen el objeto de la presente investigación. Por lo tanto, en este apartado se van a señalar aquellas líneas de cambio que, directa o indirectamente, tienen implicaciones para el desarrollo de la lengua minoritaria en el campo de la educación superior. A través del relevo generacional, se han identificado tres tendencias que se exponen a continuación, a saber, (1) el surgimiento de identidades académicas con orientación estratégica, (2) la centralidad creciente de la identidad investigadora frente a la identidad docente, y (3) el predominio del ethos académico vinculado a las identidades disciplinares de las tecnologías y las ciencias experimentales. 


\title{
4.1. Relevo generacional. Emergencia de identidades académicas con fuerte orientación estratégica
}

Es la primera tendencia identificada en el estudio empírico. En el análisis se han podido identificar dos grandes líneas de discurso que recogen, a modo de tipos ideales, diversas articulaciones entre la dimensión lingüística y la profesional. Estos discursos maestros conforman y diferencian, a su vez, a los grupos de académicos que son portadores de los mismos. El primer discurso es compartido, de un lado, por miembros de la generación pionera que abrió con sus iniciativas el campo de la educación superior a la lengua vasca en la UPV/EHU y, de otro, por miembros de una generación más joven que se reconoce continuadora de esa tradición académica y que participa de las mismas actitudes lingüisticas que aquélla, aunque en su identidad y prácticas profesionales han incorporado con mayor intensidad rasgos que corresponden a los modelos universitarios más recientes.

\begin{abstract}
Nosotros queríamos vivir en euskara. Para entonces lo teníamos claro: el euskara vale para todo o no vale para nada. Ese era nuestro principio. Nosotros debemos actualizar el euskara. Saber bien el euskara antiguo pero también adaptarlo a las nuevas condiciones. Porque estamos vivos. Si una lengua está viva, tiene capacidad para adaptarse. ¿Qué no puedo decir determinadas expresiones? $\mathrm{Si}$ las dicen en otras lenguas, nosotros también las diremos. ¿Cuál es el problema, la terminología? Pues desarrollemos la terminología, para crear un nuevo léxico. ¿Que no hemos utilizado nunca tal estructura? Pues la utilizaremos. Y si hay que inventar, se inventará. Y si hay que tomar préstamos, los tomaremos ( $1^{\text {a }}$ generación. Varón. 62 años. Ciencias Experimentales. Con publicaciones en euskara). ${ }^{1}$

Es evidente que existe una comunidad entre el profesorado, entre el profesorado que somos vascoparlante, que nos identificamos entre nosotros y que desarrollamos nuestra actividad en euskara [...] Yo creo que en el País Vasco sucede lo mismo en muchos ámbitos, en muchos sectores: identificas tu comunidad ( $2^{a}$ generación. Varón. 30 años. Ciencias de la Salud. Con publicaciones en euskara).
\end{abstract}

El segundo discurso, que se muestra con fuerza en la generación más joven, presenta evidentes discontinuidades con el de la generación pionera, tanto en lo referente a las actitudes lingüísticas como en la

\footnotetext{
${ }^{1}$ Los extractos de las entrevistas han sido traducidos del euskara para su presentación en el texto.
} 
construcción de la identidad profesional, que aparece totalmente adaptada a los requerimientos del modelo dominante.

Nosotras [los jóvenes] tenemos claro que el euskara es algo para dar clase, un instrumento. Y ya sabemos que es parte de una cultura y todo eso. Pero [...] vemos que es un instrumento para la docencia, y, sobre todo, un medio para conseguir el puesto de trabajo [...] Yo creo que ellos [los de más edad] consideran el euskara como una parte primordial de su cultura. Y nosotros 0 , por lo menos la gente de mi entorno, no. Yo solo hablo euskara en el trabajo, en la universidad. No me esfuerzo en hablar euskara en otro lugar, pero de manera consciente [...], queriendo. No quiero hablar en euskara en otros sitios que no sean la universidad. Ha sido un instrumento para conseguir el puesto de trabajo, y listo. No es que aborrezca el euskara, pero... $\left(2^{a}\right.$ generación. Mujer. 36 años. Ciencias Sociales. Sin publicaciones en euskara).

Me sorprendió que entre los jóvenes, entre las personas de mi generación, no hubiera tanto ambiente [vascoparlante]. No es lo que veo entre el profesorado de 50 años: entre ellos siempre hablan en euskara $y$, además, tienen la voluntad evidente de hablarlo. Entre la gente de mi generación, no. Hay cuatro personas, las cuatro vascoparlantes, y ves que hablan en castellano. Eso no se ve en las generaciones de más edad. Muchos de mi edad quizá lo han aprendido [el euskara] para sacar el título $\mathrm{EGA}^{2}$, mirando a que quieren entrar aquí, porque quieren dar clase $\left(2^{a}\right.$ generación. Mujer. 29 años. Ciencias Sociales. Con publicaciones en euskara).

El cambio generacional viene acompañado por el despunte de actitudes lingüísticas instrumentales frente a actitudes integrativas 0 de participación en relación a la lengua vasca, aunque esta transformación no es generalizable a todos los miembros de la generación más joven. Tales actitudes no son excluyentes entre sí, pero las actitudes integrativas tienen carácter dominante en el primer discurso y las instrumentales se muestran más abiertamente en el segundo. La práctica lingüística de los académicos vascófonos que sustentan motivaciones de participación responde a la finalidad de integrarse social y culturalmente en la comunidad vascoparlante, y de mejorar social y culturalmente la situación de aquella en el campo de la educación superior. Atienden, por tanto, a motivaciones de lealtad e identificación, que guían las prácticas lingüísticas de sus

\footnotetext{
${ }^{2}$ EGA (Euskararen Gaitasun Agiria), certificado acreditativo de conocimiento del euskara equivalente al nivel C1 del Marco Común Europeo de referencia para las lenguas.
} 
portadores en el ejercicio de las diversas tareas del rol profesional. En su caso, la dimensión lingüistica constituye un componente nuclear de la identidad personal y de la identidad profesional: es, simplemente, indisociable de ambas. En cambio, las actitudes de orientación instrumental están vinculadas a propósitos utilitarios y pragmáticos, más concretamente, a las ventajas económicas y de estatus asociadas a los puestos de trabajo como docente universitario que en los últimos años han tenido un perfil bilingüe (euskara-castellano) en la UPV/EHU.

Las dos actitudes se encuentran relacionadas con valoraciones de las lenguas en contacto, la lengua vasca y el castellano, y del inglés, y con formas diversas de construir y atribuir sentido a la carrera académica. Así, los portadores de ambas orientaciones admiten las atribuciones diferenciales de prestigio social y cultural que rigen las relaciones entre las dos lenguas oficiales en la sociedad vasca, pero mientras los primeros realizan una lectura política y conflictual de la asimetría sociolingüistica desde el punto de vista de la comunidad minoritaria -en términos de confrontación de intereses y relaciones de poder-, en los segundos hay una asunción espontánea del orden lingüístico establecido. Para estos últimos, la adquisición de la lengua vasca y su uso se sitúan en el ámbito de las opciones individuales, de los diseños vitales y profesionales de carácter estratégico que conllevan los procesos de individualización en una sociedad con multiplicidad de escenarios y lógicas de acción; en su percepción, el euskara es mayoritariamente una lengua adquirida en el sistema educativo, y su práctica está ligada actualmente al escenario laboral. Conocen la lengua vasca, en efecto, pero sus referencias sociales y culturales, sus redes de sociabilidad y de vida cotidiana, así como sus relaciones significativas en el mundo laboral, pertenecen principalmente al ámbito castellanoparlante.

Las valoraciones lingüisticas también difieren en relación a la introducción del inglés en la docencia de la educación superior: el temor y la desconfianza de unos ante la potencial reducción y disolución de los espacios docentes conquistados por la lengua minoritaria está lejos de constituir una amenaza lingüistica para los otros. Ambos grupos asumen con naturalidad la inevitabilidad del inglés como lengua de comunicación científica, pero en sus manifestaciones se vislumbra que el ámbito de la docencia sigue siendo a menudo una arena de disputa y de frágiles equilibrios entre el euskara y el castellano. 


\begin{abstract}
Yo pienso que es preciso fijarse en lo que hay fuera, y tomar como referencia países como Finlandia. Ahí utilizan la lengua propia y el inglés. El inglés para la educación superior y la lengua propia para los otros niveles [...]. Lo que ocurre es que en ese modelo el castellano está de sobra. Y en nuestra sociedad, en este momento, no se acepta ( $1^{\text {a }}$ generación. Varón. 53 años. Tecnología. Con publicaciones en euskara).

Si yo tengo que enseñar en euskara, yo no leo en castellano [...] Mis manuales están en inglés. Y yo a veces me digo "¿Cómo se dice esto en castellano?" y no tengo ni idea, y me da igual [...] Entonces, el castellano no es un instrumento de mediación $\left(1^{\mathrm{a}}\right.$ generación. Varón. 58 años. Ciencias Experimentales. Con publicaciones en euskara).
\end{abstract}

Algunos académicos con actitudes de integración en relación a la lengua vasca remarcan el carácter prescindible que debería tener el castellano en la actividad académica y científica. En este sentido, consideran conveniente la introducción del inglés siempre que la lengua internacional sustituya las funciones realizadas hasta ahora por el español $y$, en consecuencia, no reste ámbitos de uso a la lengua vasca en el campo universitario. En el caso contrario, el plurilingüismo promovido institucionalmente (con tres lenguas en la arena universitaria) se percibe como una amenaza potencial para la normalización de la lengua minoritaria.

Aquí en la facultad, por ejemplo, se han solicitado algunas sustituciones para cubrir a profesores que iban a dar clases en inglés. El profesorado que ha entrado en ese plan [plurilingüismo] ha sido mayoritariamente bilingüe [euskaracastellano], es decir, gente que hasta ahora impartían docencia en euskara. Entonces, eso tiene su parte positiva, el plurilingüismo está bien. Pero, por otro lado, existe la posibilidad de que la futura presencia del euskara disminuya. Como las asignaturas en castellano hay que mantenerlas, no disminuirán. Entonces, probablemente la cuota a pagar será en el lado del euskara. No veo que vayamos a tener una mejora evidente en un plazo corto de tiempo. Más adelante, si la relación entre el euskara y el inglés se normaliza, puede ser provechoso ( $2^{a}$ generación. Varón. 46 años. Humanidades. Con publicaciones en euskara).

La representación que construye el grupo conformado en torno a las actitudes lingüisticas de integración sobre su rol académico posee un componente activista -aunque éste se manifiesta en diversos grados y modos-. Con carácter general, puede afirmarse que la participación 
pionera de muchos docentes-investigadores vascoparlantes en la progresiva implantación del euskara como lengua de educación superior y en su modernización y actualización, así como las condiciones de excepcionalidad en las que frecuentemente han desarrollado sus tareas académicas han determinado que el compromiso lingüístico se haya incorporado como elemento esencial a la construcción de sus roles e identidades profesionales. Procesos similares han caracterizado la conformación de diversos roles en las élites intelectuales en lengua vasca (periodismo, literatura, etc.). En el campo de la educación superior, estas identidades han moldeado un ethos profesional que vincula el trabajo académico de la universidad con la responsabilidad hacia la comunidad local y su tradición de servicio público. Así, este rol no siempre se ha ajustado a las tendencias dominantes en la institución universitaria, por lo que, en la percepción de estos académicos, muchas de los trabajos y esfuerzos de carácter suplementario que ha requerido la puesta en marcha de la enseñanza en lengua vasca no les han sido profesionalmente reconocidos e institucionalmente recompensados; ni los sistemas externos de evaluación ni los indicadores vigentes de calidad de la docencia los consideran y valoran. Aún así, atribuyen sentido a la realización de un sobretrabajo carente de reconocimiento formal, debido a su orientación identitaria y su compromiso lingüístico.

Soy de las primeras docentes de esta facultad. Si miro hacia atrás, ¡cuánto hemos trabajado en tantos años! En este país hemos invertido mucho esfuerzo para lograr lo que tenemos, y la universidad tendrá que seguir trabajando. Mucho trabajo voluntario, sin remuneración. Por supuesto, cuando tienes un proyecto no mides las horas. No inviertes horas en aprender inglés y en publicar, porque tienes que hacer muchas otras cosas ( $1^{\text {a }}$ generación. Mujer. 63 años. Humanidades. Con publicaciones en euskara).

Si eres docente en euskara ves cuántas asignaturas impartes, y si eres docente en castellano vives en otra comodidad [...] Como colectivo, los docentes en euskara son más jóvenes, están más abajo en la promoción, más lejos de los puestos de autoridad, más periféricos en relación a los centros de decisión $\left(1^{a}\right.$ generación. Varón. 52 años. Ciencias Experimentales. Con publicaciones en euskara).

Desprovistos de la motivación externa comunitaria que contrarreste la racionalidad globalmente establecida, los roles académicos del segundo grupo se ajustan de manera más precisa a las exigencias 
institucionalmente dominantes, tanto en su dimensión lingüística como general. Se advierte en sus manifestaciones que las demandas de la educación superior internacionalizada comienzan a dejar su impronta en la construcción de los nuevos roles: el personal docente-investigador de la UPV/EHU, especialmente en las generaciones más jóvenes, ha interiorizado las normas y valores que orientan globalmente la actividad profesional. Esta interiorización se traduce en la autorregulación de las tareas profesionales en términos de productividad y eficiencia, que aparece además reforzada por el contexto social de precariedad laboral. En esta línea, el uso de la lengua minoritaria más allá de la actividad estrictamente docente de la impartición de clases (que para algunos deja de incluir la producción de material docente, la modernización del corpus lingüistico, la actividad de divulgación, y la propia capacitación y desarrollo de las competencias lingüisticas del docente) pierde sentido en la cultura de la gestión del rendimiento. Así, a los ojos de muchos jóvenes académicos, la realización de tesis y la investigación y comunicación científicas en lengua minoritaria devienen actividades excesivamente gravosas para una carrera académica que, ya sin cargas añadidas, debe superar múltiples obstáculos en un entorno de creciente competitividad. Las estrategias lingüisticas puestas en marcha revelan el sentido que han conferido a su trayectoria profesional y las consecuencias derivadas para la lengua minoritaria.

No, no, no se me ha pasado por la cabeza [hacer la tesis en euskara] [...] Ahora estoy en otras cosas. Pronto saldrá mi plaza como agregada [...] No puedo con todo, tenemos que ir a congresos, escribir artículos, ponencias, las clases. Cada curso tengo asignaturas distintas [...] No puedo hacerlo todo bien. ¡Encima en euskara! ( $2^{a}$ generación. Mujer. 41 años. Ciencias Sociales. Sin publicaciones en euskara).

He tenido claro desde el principio hacerla [mi trayectoria académica] en castellano [...] Yo lo veo a mi alrededor. Algunos tienen el compromiso. Yo tengo algún conocido que hace esto: "yo publico un tercio en euskara". Pero, claro, son muy racionales, y lo hacen por militancia. Lógicamente, ese tercio va a tener menor rentabilidad que los otros dos tercios. $y$, normalmente, la persona que está en precario [...] Y eso es lo que ocurre con el euskara. Si yo tuviera ganas de publicar en euskara, tengo pocos incentivos para hacerlo. Está claro. ( $2^{a}$ generación. Varón. 40 años. Ciencias Sociales. Sin publicaciones en euskara). 
En las manifestaciones de los docentes vascófonos de la segunda generación que revelan actitudes instrumentales hacia el euskara, se percibe una asunción tácita del modelo de universidad vigente en la actualidad y, en consecuencia, una interiorización más naturalizada de la cultura que ha traído consigo la internacionalización. Así, la construcción de las identidades académicas de dichos docentes-investigadores se ha ajustado al nuevo modelo y a sus requerimientos. Por el contrario, se vislumbran actitudes más críticas sobre los nuevos patrones dominantes en la educación superior entre aquellos que comparten actitudes lingüísticas de integración en relación a la lengua vasca.

Somos un gestor, a favor de la ciencia, a favor de la empresa. En eso se lucha mucho, pero de cara a la sociedad [...] no somos una referencia [...]. Ha entrado la meritocracia, la productividad, la productividad científica en inglés. Entonces, nos es igual ser docente en euskara, en inglés, en alemán... ( $2^{a}$ generación. Varón. 48 años. Ciencias de la Salud. Con publicaciones en euskara).

La perspectiva crítica adquiere especial intensidad entre los académicos de mayor edad, que proceden de experiencias intelectuales y vitales diferentes. En el discurso de estos últimos, la cuestión de la lengua minoritaria se enmarca y se significa constantemente, más allá de la opción individual, en un marco social más amplio, que es también el marco de los modelos universitarios posibles.

\subsection{Centralidad creciente de la identidad investigadora frente a la identidad docente}

La tensión que se está produciendo entre los mundos de la docencia y la investigación en la conformación de los roles académicos, hace que su construcción oscile entre estas dos áreas de actividad que generan en la universidad actual exigencias contradictorias y recompensas diferenciadas. En las primeras generaciones de académicos vascófonos de la UPV/EHU, la dimensión docente ha sido muy relevante. Esta transcendencia no puede desligarse del sentido prioritario que se le ha atribuido al proyecto de implantación de la enseñanza en euskara, en cuyo proceso se han sentido personal y profesionalmente implicados. En las generaciones más jóvenes, sin embargo, se parte de una percepción en la que la enseñanza en euskara está ya institucionalizada en todos los niveles educativos, también en el superior, aunque sea parcialmente. Además, en este sector de menor edad se afianza una identidad profesional que 
contempla la investigación como componente dominante de la actividad profesional. Se produce asi un ajuste entre los requerimientos institucionales, que incentivan la investigación sobre la docencia, y los valores profesionales.

Por un lado, si son docentes jóvenes, no tienen ese afecto hacia el euskara. Han recibido el euskara desde la ikastola, pero no por propia voluntad, sino a saber por qué. Entonces falta ese afecto. Por otro lado, como nos insisten tanto en que nos internacionalicemos, los grupos de investigación trabajan en inglés, y en otras lenguas, en lenguas extranjeras. Si se han introducido de cabeza en el trabajo de investigación, no pueden dedicarle el tiempo necesario a la docencia. Si han orientado la profesión hacia la investigación, aunque ejerzan la docencia, queda poco tiempo para la docencia. Y si hay poco tiempo para la docencia, menos aún para producir material [docente] en euskara. ( $2^{\text {a }}$ generación. Varón. 42 años. Tecnología. Con publicaciones en euskara).

Son dos mundos totalmente distintos, totalmente. Hay un camino, el de la docencia, por decirlo de alguna manera, y otra trayectoria, la de la investigación. Y, a menudo, somos ambivalentes. Yo, al menos, lo soy [...] Tienes dos profesiones. Eres docente [...] Y, por otro lado, eres investigador y realizas esa investigación en castellano. Y no siento que exista ninguna contradicción. Lo estoy diciendo en tanto que sujeto no militante. Yo, en ese sentido, soy bastante cínico. Sí, soy cínico, no siento ninguna obligación moral hacia el euskara o el mundo vascoparlante. Entonces, en ese sentido, soy un sujeto muy posmoderno. Tengo una doble moralidad y me siento muy a gusto, enseñando en euskara e investigando en castellano $\left(2^{\text {a }}\right.$ generación. Varón. 43 años. Ciencias Sociales. Sin publicaciones en euskara).

Como consecuencia de las exigencias institucionales en torno al diseño de la carrera académica, en la conformación de la identidad profesional de los docentes investigadores más jóvenes se aprecia que la tensión docenciainvestigación se ha decantado claramente a favor de la investigación. A esta opción prioritaria se le añade, además, la interiorización de una lógica de eficiencia productivista, la exaltación de los valores pragmáticoeconómicos y la consecuente renuncia a objetivos profesionales que no impliquen rentabilidad económica o simbólica.

Aqui tenemos que trabajar sin fanatismos ni maximalismos. Si hay posibilidad de contratar a profesionales punteros, aunque no sepan euskara, si son investigadores de primera, eso le hará 
un gran favor a la universidad. Gracias a eso, la universidad ascenderá en todos los rankings. Ganará proyectos que reciben mucho dinero. Hay que jugar con flexibilidad, siempre. $\left(2^{a}\right.$ generación. Varón. 31 años. Ciencias Experimentales. Sin publicaciones en euskara).

En los últimos años existe una gran presión. Si. Yo pienso que estos últimos años la ANECA, UNIQUAL y similares nos empujan sobre todo a la investigación. Aunque nos contratan para la docencia, nos pagan por la investigación [...] Le dedicamos tiempo a la investigación, en inglés, y dejamos de lado el euskara. Dejamos de lado la labor de producir material en euskara ( $2^{a}$ generación. Varón. 42 años. Tecnología. Con publicaciones en euskara).

A pesar de que la investigación es estimulada en mayor grado que la docencia, los académicos vascófonos consideran que la incentivación de la actividad investigadora no tiene reflejo en el impulso de la lengua minoritaria en este ámbito. Por un lado, por la inexistencia de instrumentos de medición para las lenguas minoritarias: la investigación publicada en euskara, sometida a las normas de agencias externas de evaluación, queda desprovista de todo valor. Por otro lado, porque el grado de especialización de ciertas disciplinas exige, como destinataria de la comunicación científica, una masa crítica potencial que la comunidad lingüistica minoritaria no puede ofrecer.

A nivel competitivo no se valora [la producción en euskara]. Pienso que existe un concepto que ha producido un perjuicio, y es el del impacto. Y el impacto está bien para valorar lo que se produce en inglés, sobre todo en química, bioquímica, biología, medicina y física. Son éstos los que inventaron el concepto de impacto. Pero, no se adecúa bien a otros campos. Valorar la investigación únicamente con este criterio se queda muy corto. y, por ejemplo el euskara se queda fuera. ( $1^{\text {a }}$ generación. Varón. 53 años. Tecnología. Con publicaciones en euskara).

Esta universidad utiliza los parámetros de otras universidades para medir la investigación, sexenios y similares. Entonces, tu puedes enviar a un sexenio algo en euskara [...] Pero si no es del ámbito de la filología vasca [...] no se toma en consideración [...] Aquellos que hemos decidido producir en euskara tenemos que realizar un trabajo extra. Y debemos publicar nuestros trabajos en inglés, en castellano, y en otras lenguas [...] La persona que opta por el euskara está actuando contra sí mismo en esa promoción. Está muy claro. Yo he publicado algunos libros [en euskara]. Este es el último [lo muestra]. Pasé un par de años escribiéndolo. Voy a publicar un resumen en una revista [en 
castellano], y valdrá mucho más ese resumen de la revista que el libro [...] Yo podía haber escrito el artículo, sin más [...] Publicar en euskara, en principio, es un gran obstáculo para el desarrollo del curriculum del docente $\left(1^{\text {a }}\right.$ generación. Varón. 49 años. Humanidades. Con publicaciones en euskara).

No obstante, en relación a la comunicación científica y la consideración del inglés como su portador exclusivo, se evidencian en las entrevistas actitudes muy diferenciadas entre los académicos de las ciencias experimentales y las tecnologías, y los académicos de las ciencias sociales y humanas. En tanto que los primeros atribuyen a la lengua minoritaria una única función divulgativa - la función científica queda reservada al inglés-, los segundos, especialmente los pertenecientes a la primera generación, estiman que la investigación científica en sus disciplinas puede y debe ser comunicada a la sociedad en su lengua de referencia. Aún más, consideran parte de su identidad académica el compromiso con la sociedad de pertenencia y se arrogan el papel de intelectuales, dentro de una representación que contempla la universidad no sólo como entidad productora de conocimiento sino también como institución con responsabilidades éticas hacia la sociedad civil en la que está arraigada, hacia su desarrollo y su bienestar. Precisamente, la responsabilidad lingüística aparece integrada en esta concepción general.

[El lugar del euskara en el ámbito científico] es la divulgación, la divulgación culta ( $1^{\text {a }}$ generación. Varón. 58 años. Ciencias Experimentales. Con publicaciones en euskara).

Todos los artículos los he publicado en inglés. Solo en inglés. Nuestras publicaciones deben de estar en el famoso ISI [...] Por ese lado, yo no hago militancia [a favor del euskara]. (2a generación. Varón. 31 años. Ciencias Experimentales. Sin publicaciones en euskara).

No se publica en euskara porque sí, por hacerlo en euskara, sino porque queremos darle un lugar a la universidad en su sociedad. Existe ahí implícitamente un valor que debe ser cultivado. No es solo la lengua ( $1^{\text {a }}$ generación. Mujer. 63 años. Humanidades. Con publicaciones en euskara).

De este modo, existe una clara diferenciación entre los académicos de las dos grandes ramas del conocimiento (ciencias experimentales y tecnologías, por un lado, y ciencias sociales y humanas, por otro), que desarrollan actitudes y prácticas distintas en relación a la comunicación científica, y a las lenguas en que debe desarrollarse ésta. 


\subsection{Preeminencia del ethos académico ligado a las identidades disciplinares de las tecnologías y las ciencias experimentales}

La tendencia apuntada se desarrolla de forma paralela a la posición crecientemente marginal de las ciencias sociales y las humanidades en el campo de la educación superior. Los valores de la economía del conocimiento y la incorporación como núcleo dominante de la identidad académica de la lógica científico-positivista de las ciencias y las tecnologías han traído consigo la pérdida de valor simbólico de las disciplinas consideradas "no productivas" - ciencias sociales y humanas-. En el plano cognitivo este dominio se traduce en las pretensiones de producción de un saber pragmático, estandarizado y universalizable, cada vez más desligado de las motivaciones sociales o humanas y de su contexto de producción; en el plano lingüístico, se refleja en el monopolio del inglés en su comunicación, y en la atribución a la lengua franca de un papel supuestamente neutro e instrumental. En efecto, la concepción de la neutralidad lingüística, es decir, la suposición de que la lengua es un mero instrumento portador que no conforma ni produce efectos en los conocimientos producidos, claramente dominante en las ciencias experimentales y la tecnología, se intensifica y refuerza al converger con una práctica lingüistica que, de facto, erige al inglés como única lengua legitimada de comunicación científica universal. Desde la perspectiva que domina la tradición disciplinar de algunos académicos de ciencias experimentales y de tecnología, la lengua minoritaria no tiene un estatus de legitimidad en la producción científica, sino es el espacio residual asignado a la divulgación y -casi en el mismo nivel de degradación- a la producción de las ciencias sociales y humanidades. Lo que contrasta con el valor sustantivo conferido a la lengua por parte de los profesionales de estas últimas áreas de conocimiento.

En ciencias el inglés es la lengua principal. En otras áreas de conocimiento quizá las cosas serán distintas. Sin duda. Yo creo que si se hace un trabajo sobre el euskara, a nivel lingüistico, lo más lógico es que se publique en euskara. No es lo mismo. Si alguien hace un trabajo sobre el euskara como lingüista... y también en Sociología, si alguien hace Sociología en el País Vasco, ese trabajo debe ir en euskara. Yo pienso que sí. Los más interesados son los vascos. Creo que esa posibilidad es distinta en los temas científicos y en el campo de las letras [sic]. Creo que es distinto. En nuestra facultad la lengua científica es el inglés. Por ese lado, considero que el castellano y el euskara están al 
mismo nivel $\left(1^{\text {a }}\right.$ generación. Mujer. 59 años. Ciencias Experimentales. Con publicaciones en euskara).

En Ciencias Naturales son muy instrumentales. Yo, por el contrario, no puedo pensar que en Ciencias Sociales la lengua pueda ser utilizada como simple instrumento. La lengua tiene una vida y es un recurso tremendo. En las ciencias Sociales se le debe dar importancia a la propia lengua, porque las formas de decir son imprescindibles. Los de Ciencias Naturales son muy instrumentales, y utilizan la lengua, por decirlo de alguna manera, porque tienen que expresar cosas muy proposicionales, muy simples, y la mayor parte de las veces utilizan los números ( $2^{a}$ generación. Varón. 43 años. Ciencias Sociales. Sin publicaciones en euskara).

En este escenario, la remodelación de las jerarquías simbólicas del mundo científico-académico debilita la posición de los académicos de las disciplinas sociales y humanas $y$, además, pone en duda su concepción de universidad y la forma de entender el rol profesional dentro de la institución académica. En la percepción de los académicos vascófonos de las ciencias sociales y humanas de la UPV/EHU, su aportación intelectual cobra sentido no sólo porque puede ser comunicada en lengua minoritaria a la sociedad de referencia - a la vez que en otras lenguas-, sino porque con sus saberes expertos nutre de argumentos de legitimación las luchas simbólicas que se desarrollan en toda situación de contacto lingüístico, y que afectan el estatus y el prestigio de la lengua minoritaria. Es por ello por lo que su pérdida de centralidad en las estructuras universitarias supone también el declive de la idea de una producción académica vinculada a los intereses de la sociedad local $y$, por ende, de una profesión con obligaciones y compromisos hacia la comunidad lingüistica minoritaria. Mientras que el discurso del académico de las ciencias experimentales o de las tecnologías, aún mostrándose favorable al desarrollo de la lengua minoritaria, circunscribe el uso de ésta al trabajo estrictamente académico en el sentido restringido del término, el discurso proclive a la asignación de una función intelectual (es decir, política) a los académicos vascoparlantes aparece con mayor nitidez en los docentes investigadores de las disciplinas sociales/humanas y parece intensificarse en las personas de mayor edad.

¿Por qué debemos usar el euskara [en la universidad]? ¿Qué hay detrás de eso? Pues, el vínculo con la sociedad. Si el País Vasco tiene que ser país vasco. El estatus que nosotros le queremos otorgar, el sitio que le queremos dar, no va a venir únicamente de los trabajos que realicemos a nivel internacional $\left(1^{\mathrm{a}}\right.$ 
generación. Mujer. 63 años. Humanidades. Con publicaciones en euskara).

El análisis de los discursos apunta a una preeminencia y valoración creciente en el mundo académico de disposiciones profesionales propias de las disciplinas consideradas como productivas para el mercado económico. El modelo de identidad académica que integra entre sus funciones profesionales cometidos intelectuales y de influencia crítica en el escenario social y político posee escasa cabida en ese modelo. Y ello origina un debilitamiento paralelo de las disposiciones académicas orientadas al activismo lingüístico en el campo de la educación superior.

\section{CONCLUSIONES}

La globalización ha penetrado con fuerza en la institución universitaria en los últimos años. Y ha tenido efectos importantes en diversos ámbitos, tanto a nivel académico como profesional. Balfour (2007) sugiere que la planificación lingüística en las instituciones de la educación superior puede ser mediatizada, conformada, y también distorsionada, por las dinámicas derivadas de la globalización. En este trabajo se ha pretendido llevar esta aseveración al estudio de las identidades académicas, un ámbito que aparentemente parece no estar en relación directa con las políticas lingüisticas. Con este propósito, se han identificado algunas de las tendencias de cambio que se están produciendo en los roles e identidades profesionales de distintas generaciones de docentes-investigadores que desarrollan su trabajo en lengua minoritaria en una universidad multilingüe (la UPV/EHU), entre otros: la emergencia de fuertes orientaciones estratégicas que guian el desempeño de las tareas profesionales, la alteración de la tabla de valores académicos tradicionales y el subsiguiente reposicionamiento simbólico de dichas tareas fundamentalmente la docencia y la investigación-, así como el predominio de una concepción de universidad más ligada a los requerimientos de la economía global que a las necesidades de la sociedad local. Tales cambios, ligados a los procesos de globalización e internacionalización de la educación superior, están relacionados con las metamorfosis culturales derivadas de la integración de la universidad en la economía del conocimiento. Más concretamente, con la implantación de modelos gerencialistas y de sistemas de auditoría orientados hacia los valores de la eficiencia y el rendimiento. 
El estudio de la articulación entre la dimensión lingüística y profesional que realizan los académicos de la UPV/EHU a la hora de diseñar, construir y representar su carrera profesional ha permitido visibilizar las implicaciones de agenda oculta que tiene la nueva estructura cultural de la educación superior internacionalizada en el ámbito lingüístico. En efecto, las orientaciones estratégicas e instrumentales que rigen las nuevas identidades académicas constituyen una política lingüistica encubierta que actúa en detrimento de las lenguas más débiles con presencia en la educación superior. Tales orientaciones resultan tanto más eficaces cuanto más integradas aparecen en las diversas expresiones culturales del entramado meritocrático, y tanto más influyentes cuanto más inadvertido queda su carácter sistémico y constrictivo, ya incorporado en las disposiciones profesionales de las nuevas generaciones de académicos.

Estas consideraciones revelan que la opción de las universidades europeas con lenguas minoritarias -entre los que se encuentra la UPV/EHU (Cots, Lasagabaster y Garrett, 2012) - a favor de un modelo de competitividad económica, no es una opción irrelevante para la lengua minoritaria. La UPV/EHU se ha caracterizado desde los años 80 por su compromiso institucional para la promoción de la lengua vasca como lengua vehicular en la educación superior. A tal fin ha puesto en marcha sucesivos programas para su integración en el campo docente y científico. La preparación y capacitación lingüistica del profesorado ha formado parte del proyecto. Pero, tales iniciativas no parecen ser totalmente convergentes con los modelos vigentes de internacionalización que se han adoptado. Hay un contexto de valores y requerimientos institucionales contradictorios que guían el diseño de las identidades de los académicos, y las fuerzas del modelo hegemónico de internacionalización no siempre son coincidentes con las políticas activas a favor de la lengua minoritaria en el diseño de la carrera académica; la mutación de las identidades académicas de los académicos vascófonos, especialmente visible a través del relevo generacional del personal docente-investigador, muestra la fuerza reguladora que tiene el modelo económico en las opciones lingüisticas implícitas en las tareas docentes e investigadoras.

Por último, el análisis pone de manifiesto la necesidad que tienen las instituciones de la educación superior con lenguas minoritarias de iniciar un debate profundo sobre los posibles modelos de internacionalización, sobre las formas de entender y reconciliar las dinámicas globales y locales, y sobre la conveniencia de optar por aquéllos que sean respetuosos con la 
diversidad cultural y lingüistica. La internacionalización no tiene necesariamente un sentido único; las universidades han sido internacionales de muchas maneras, como recuerda Altbach (2004). El modelo de internacionalización está relacionado con la definición de la identidad de la propia universidad, con la formulación de sus objetivos y de su responsabilidad social (Bull, 2012). Sólo ese debate previo puede clarificar la diversidad de consecuencias lingüísticas y de identidades académicas implicadas en cada modelo.

\section{BibLiOgRAFÍA}

Alcón, E. (2012). El inglés en un marco de actuación multilingüe. In E. Alcón $\&$ F. Michavila (Eds.), La Universidad Multilingüe (pp. 167-183). Madrid: Tecnos.

Altbach, P. G. (2004). Globalisation and the university: myths and realities in an unequal world. Tertiary Education and Management, 10, 3-25.

Altbach, P.G., Reisberg, L., \& Rumbley, L.E. (2009). Trends in Global Higher Education: Tracking an Academic Revolution. Paris: UNESCO

Ammon, U. (2012). Linguistic inequality and its effects on participation in scientific discourse and on global knowledge accumulation. Applied Linguistics Review, 3(2), 333-355. http://dx.doi.org/10.1515/applirev2012-0016. Última consulta: 22/09/2016.

Archer, L. (2008). The new neoliberal subjects? Young/er academics' constructions of professional identity. Journal of Education Policy, 23(3), 265-285.

Artetxe, K. (2011). Euskararen aurrerabidea zientzia eta goi mailako esparruan. Azterketa bibliometrikoa. Vitoria-Gasteiz: Eusko Jaurlaritza-Gobierno Vasco.

Balfour, R. J. (2007). University language policies, internationalism, multilingualism, and language development in the UK and South Africa. Cambridge Journal of Education, 37, 35-49.

Ball, J. S., \& Youdell, D. (2007). Hidden Privatisation in Public Education. London: University of London.

Barnett, R., \& Di Napoli, R. (2008). Changing Identities in Higher Education. London: Routledge.

Bauman, Z. (2004). Identity. Cambridge: Polity Press. 
Bull, T. (2012). Against the mainstream: universities with an alternative language policy. International Journal of the Sociology of Language, 216, 55-74.

Burton-Jones, A. (2001). Knowledge Capitalism: Business, Work and Learning in the New Economy. Oxford: Oxford University Press.

Canaan, J.F., \& Shumar, W. (Eds.). (2008). Structure and Agency in the Neoliberal University. New York: Routledge.

Cenoz, J. (2012). Bilingual Educational Policy in Higher Education in the Basque Country. Language, Culture and Curriculum, 25(1), 41-55.

Churchman, D., \& King, S. (2009). Academic practice in transition: hidden stories of academic identities. Teaching in Higher Education, 14(5), 507516.

Cots, J., Lasagabaster D., \& Garrett, P. (2012). Multilingual policies and practices of universities in three bilingual regions in Europe. International Journal of the Sociology of Language, 216, 7-32.

Cots, J. (2013). Introducing English-Medium Instruction at the University of Lleida, Spain: Intervention, Beliefs and Practices. In A. Doiz, D. Lasagabaster \& J. M. Sierra (Eds.), English-Medium Instruction at Universities (pp. 106-128). Bristol: Multilingual Matters.

Cots, J., Llurdá, E., \& Garrett, P. (2014). Language policies and practices in the internationalisation of higher education on the European margins: an introduction. Journal of Multilingual and Multicultural Development, 35, 311-317.

Di Napoli, R. (2010). Identidades académicas y gestión: ¿una misión imposible?. In J. Rué \& L. Lodeiro (Eds.), Equipos docentes y nuevas identidades académicas en Educación Superior (pp. 189-201). Madrid: Narcea.

Doiz, A., Lasagabaster, D., \& Sierra, J.M. (2013). English as L3 at a Bilingual University in the Basque Country, Spain. In A. Doiz, D. Lasagabaster \& J. M. Sierra (Eds.). English-Medium Instruction at Universities. Global Challenges (pp. 84-105). Bristol: Multilingual Matters.

Dubet, F. (2006). El declive de la institución. Profesiones, sujetos e individuos en la Modernidad. Barcelona: Gedisa.

Dubet, F. (2009). Le travail des sociétés. París: Seuil.

Faure, S., Soulié, Ch., \& Millet, M. (2011). Enquête exploratoire sur le travail des enseignants-chercheurs. http://www2.univparis8.fr/sociologie/fichiers/ soulie2005a.pdf. Última consulta: 20/09/2016. 
Gazzola, M. (2012). The linguistic implications of academic performance indicators. International Journal of the Sociology of Language, 216, 131156.

Gordon, G., \& Whitchurch, C. (Eds.). (2010). Academic and Professional Identities in Higher Education: The Challenges of a Diversifying Workforce. New York: Routledge.

Gutiérrez-Solana, F., Ruiz, A., \& Etayo, F., (2012). Las lenguas en la actividad investigadora. In E. Alcón \& F. Michavila (Eds.). La Universidad Multilingüe (pp. 37-53). Madrid: Tecnos.

Hamel, R.E. (2013). El campo de las ciencias y la educación superior entre el monopolio del inglés y el plurilingüismo. Trabalhos em Linguistica Aplicada, 52(2), 1-31.

Harris, S. (2005). Rethinking academic identities in neo-liberal times. Teaching in Higher Education, 10(4), 421-433.

Hernández, J., Delgado-Gal, A., \& Pericay, X. (Eds.), (2013). La universidad cercada. Barcelona: Anagrama.

Iglesias, J.I., \& Salaburu, P. (2003). Unibertsitatea eta euskal gizartea, gaur. Iruñea-Pamplona: Pamiel,.

Kehm, B.M., \& Teichler, U. (Eds.), (2013). The Academic Profession in Europe: New Tasks and New Challenges. Netherlands: Springer.

Larrinaga, A. (2007). Kulturgileak eta kulturaren esparrua II. Euskal kulturgileak euskalgintza mugimenduaren eraikuntzan. Donostia: Utriusque Vasconiae.

Lasagabaster, D. (2012a). Programes de multilingüisme al País Basc: objetius, assoliments i actituds lingüistiques. In A. Bos i Solé (Ed.). Les fronteres del multilingüisme. Una visió actual i pluridisciplinar sobre el multilingüisme en la societat contemporània (pp. 29-54). Barcelona: UOC.

Lasagabaster, D. (2012b). Políticas multilingües y sus resultados en el ámbito universitario europeo. In E. Alcón \& F. Michavila (Eds.). La Universidad Multilingüe (pp. 111-129). Madrid: Tecnos.

Lasagabaster, D. (2012c). El papel del inglés en el fomento del multilingüismo en la universidad. ELIA, 12, 13-44.

Lynch, K. (2011). International league tables and rankings in higher education: an appraisal. In I. Elliott, M. Murphy, A. Payeur \& R. Duval (Eds.). Change in Higher Education and Globalisation(pp. 19-44). Bruxelles: De Boeck.

Marginson, S., \& Considine, M. (2000). The Enterprise University. VictoriaOakleigh: Cambridge University Press. 
Michavila, F. (2012). La universidad multilingüe. In E. Alcón \& F. Michavila (Eds.). La Universidad Multilingüe (pp. 19-37). Madrid: Tecnos.

Michavila, F., \& Martínez, J. (Eds.). (2004). La profesión de profesor de universidad. Madrid: Comunidad de Madrid, Cátedra UNESCO de Gestión y Política Universitaria, Universidad Politécnica de Madrid.

Montané, A., \& Sánchez de Serdio, A. (2011). Sujeto a reforma: la transformación de la identidad docente en la Educación Superior. RASE 4(1), 73-88.

Ninnes, P., \& Hellstén, M. (Eds.), (2011). Internationalizing Higher Education: Critical Explorations of Pedagogy and Policy. Dordrecht: Springer.

Sabaté-Dalmau, M. (2016). The Englishation of higher education in Catalonia: a critical sociolinguistic ethnographic approach to the students perspectives. Language, Culture and Curriculum, http://dx.doi.org/10.1080/07908318.2016.1153108. Última consulta: 22/09/2016.

Sancho, J.M., Creus, A., \& Padilla, P. (2010). Docencia, investigación y gestión en la Universidad: una profesión tres mundos. PRAXIS Educativa, XVI(14), 17-34.

Schleider, A. (2006). The Economics of Knowledge. Why Education is key for Europe's success. Brussels: OECD, The Lisbon Council Policy Brief.

Sennet, R. (1998). The Corrosion of Character: The Personal Consequences of Work in the New Capitalism. New York: N.W. Norton \& Company.

Shapper, J., \& Mayson, S. (2011). Managerialism, Internationalization, Taylorization and the Deskilling of Academic Work. In P. Ninnes \& M. Hellstén (Eds.). Internationalizing Higher Education: Critical Explorations of Pedagogy and Policy (pp. 181-197). Dordrecht: Springer,

The Council of the European Union (2009). Council Conclusions of 12 May 2009 on a strategic framework for European cooperation in education and training («ET 20120»). http://eur-lex.europa.eu/legalcontent/EN/TXT/?uri=URISERV\%3Aef0016. Última consulta: 22/09/2016.

Truchot, C. (2010). Questions de langues dans l'internationalisation de l'enseignement supérieur en Europe. Éducation et Societés Plurilingues, 29, 85-97.

UPV/EHU (2011). UPV/EHUko Estatutuak. Estatutos de la UPV/EHU. Leioa: Servicio Editorial de la Universidad del País Vasco. http://www.ehu.eus/documents/3026289/31. Última consulta: 22/09/2016. 
UPV/EHU (2013a). UPV/EHU en cifras. http://www.ehu.eus/zenbakitan/es. Última consulta: 22/09/2016.

UPV/EHU (2013b). UPV/EHUko Euskararen II. Plan Gidaria (2013-2017). http://www.ehu.eus/documents/1814721/2017708/plan_gidaria.pdf. Última consulta: 22/09/2016.

Vila, F. X., Bretxa, V., \& Comajoan, LI. (2014). ¿En qué lenguas se hace la ciencia? La gestión del multilingüismo en el Parc Cientific de Barcelona. Revista Internacional de Organizaciones, 13, diciembre, 111-134.

Villarruel, M. (2012). Identidad docente y exigencia académica: encuentros y desencuentros con la realidad social. Perspectiva Educacional, 51(1), 2944. 